Western University

Scholarship@Western

Department of Economics Research Reports

Economics Working Papers Archive

1982

\title{
A Lagrange Multiplier Test of the Restrictions for a Simple Rational Expectations Model
}

\author{
Allan W. Gregory \\ Michael R. Veall
}

Follow this and additional works at: https://ir.lib.uwo.ca/economicsresrpt

Part of the Economics Commons

Citation of this paper:

Gregory, Allan W., Michael R. Veall. "A Lagrange Multiplier Test of the Restrictions for a Simple Rational Expectations Model."

Department of Economics Research Reports, 8216. London, ON: Department of Economics, University of Western Ontario (1982). 
ISSN: $\quad 0318-725 \mathrm{X}$

ISBN: $0-7714-0367-4$

RESEARCH REPORT 8216

A LAGRANGE MULTIPLIER TEST OF THE RESTRICTIONS FOR A SIMPLE RATIONAL EXPECTATIONS MODEL

by

Allan W. Gregory and

Michael R. Veall

\begin{abstract}
The Lagrange multiplier test of the restrictions for a simple but common class of rational expectations models is developed. The corresponding test statistic is shown to be reasonably easy to calculate in most practical examples and Monte Carlo evidence suggests it performs as well as the Wald and likelihood ratio tests in small samples.
\end{abstract}

$$
\text { Ju1y, } 1982
$$

The authors would like to thank Glenn MacDonald and Aman U1lah.

Jル 281992

University of Western Ontario 


\section{A Lagrange Multiplier Test of the Restrictions for a Simple Rational Expectations Model \\ Allan W. Gregory and Michael R. Veall}

\section{Introduction}

Discussion of the testing of the restrictions implied by the rational expectations hypothesis has focussed on the Wald (W) and likelihood ratio (LR) principles of testing (see, for example, Hoffman and Schmidt, 1981 and Revankar, 1980). For the most part, this literature has ignored the asymptotically equivalent Lagrange multiplier (IM) test.

The purpose of this paper is to derive the IM test for a simple but common class of rational expectations models. The test involves summing $\mathrm{R}^{2}$ 's from a set of auxiliary linear regressions using the residuals from restricted estimation and is therefore quite easy to calculate. In addition, we examine the small sample properties of the IM test using a Monte Carlo experiment which follows the design of Hoffman and Schmidt (1981).

2. The model

Consider the following rational expectations model of Hoffman and Schmidt (hereafter referred to as $\mathrm{H}-\mathrm{S})^{1}$ :

$$
y_{t}=\sum_{i=1}^{K} \beta_{i} x_{t i}+\lambda E_{t-1} y_{t}+\epsilon_{t}
$$

where $y_{t}$ is the dependent variable, $X^{\prime}$ 's are exogenous variables, $E_{t-1}$ is the mathematical expectations operator indicating the rational expectation of $y_{t}$ conditional on the information available at time $t-1$, $\beta_{i}(i=1, \ldots, K)$ and $\lambda$ are unknown parameters and $\epsilon_{t} \sim N I D\left(0, \sigma_{\epsilon}^{2}\right)$. It is also as sumed that $\operatorname{plim}\left(\sum \frac{X_{t i} \epsilon_{t-r}}{N}\right)=0$ for any integer $r$ and $i=1, \ldots, K_{\bullet}^{2}$ 
Taking expectations of equation (1) and solving yields:

$$
y_{t}=\sum_{i=1}^{K} \beta_{i} x_{t i}+\lambda(1-\lambda)^{-1} \sum_{i=1}^{K} \beta_{i} E_{t-1} x_{t i}+\epsilon_{t}
$$

where $\lambda \neq 1$. In order to complete the model, it is necessary to specify the process generating the exogenous variables. It is common to assume that these variables follow some autoregressive process as in Wallis (1980). For this example, it is assumed that each exogenous variable follows a univariate autoregressive process of order $Q<N$ :

$$
x_{t i}=\sum_{j=1}^{Q} \gamma_{i j} x_{t-j, i}+u_{t i} \quad i=1, \ldots, k
$$

where $u_{t i} \sim \operatorname{NID}\left(0, \sigma_{i}^{2}\right), \operatorname{plim}\left(\sum \frac{u_{t i} u_{t-r_{2} s}}{N}\right)=0$, and $\operatorname{plim}\left(\Sigma \frac{x_{t-i} i^{u_{t i}}}{t}\right)=0$ for any integer $r, j=1, \ldots, Q$ and $i, s=1, \ldots, k$, $i \neq s$. For the results below, it is not necessary that the order of the autoregressive process be the same for each equation; nor is it necessary to restrict the analysis to univariate processes, provided that an analogous set of orthogonality conditions to those given above hold for the vector autoregressive processes.

Applying the expectation operator to equation (3) and substituting this into (2) gives:

$$
y_{t}=\sum_{i=1}^{K} \beta_{i} x_{t i}+\lambda(1-\lambda)^{-1} \sum_{i=1}^{K} \sum_{j=1}^{Q} \beta_{i} \gamma_{i j} x_{t-j, i}+\epsilon_{t}
$$

Notice that the $\gamma_{i j}$ 's may be obtained directly from equation ( 3 ). In equation (4) there are $K Q+K$ variables but only $K+1$ 'free' parameters implying KQ-1 restrictions. The unrestricted equation corresponding to equation (4) is:

$$
y_{t}=\sum_{i=1}^{K} \sum_{j=0}^{Q} \alpha_{i j} x_{t-j, i}+\epsilon_{t}
$$


It is straightforward to verify the restrictions on the parameters of equation (3) and equation (5) are:

$$
H_{0}: \alpha_{i j} / \alpha_{i o} \gamma_{i j}=\alpha_{m n} / \alpha_{m o} \gamma_{m n} \quad i, m=1, \ldots, K \text { and } j, n=1, \ldots, Q \text {. }
$$

Equation (6) gives $\mathrm{KQ}-1$ independent overidentifying restrictions.

3. Testing the restrictions

The restrictions on equations (3) and (5) given by equation (6) may be tested using IR, $W$ and IM tests. For purposes of comparison, the IR and W tests discussed by $\mathrm{H}-\mathrm{S}$ for this model are given below and then the LM test is developed.

The LR test requires: (i) estimating the unrestricted model (equations (3) and (5)) by ordinary least squares and summing the values of the $\log$ of the likelihood functions for these $\mathrm{k}+1$ regressions; (ii) estimating the restricted system (equations (3) and (4)) by non-linear methods and obtaining twice the difference of the sum of the likelihoods from (i) and the restricted value from (ii). This statistic is asymptotically distributed as a $\chi^{2}(\mathrm{KQ}-1)$ under $\mathrm{H}_{\mathrm{O}}$. In practice this presents no difficulty.

The $\mathrm{W}$ test involves estimating the unrestricted model and forming the quadratic:

$$
h(\hat{\theta})^{\mathrm{T}}\left[\hat{\mathrm{H}}_{\theta} \hat{\mathrm{V}}(\hat{\theta}) \hat{\mathrm{H}}_{\theta}^{\mathrm{T}}\right]^{-1} \mathrm{~h}(\hat{\theta}) \stackrel{a}{\sim} \chi^{2}(\mathrm{KQ}-1)
$$

under $\mathrm{H}_{\mathrm{O}}$, where $\theta$ is the unrestricted parameters of equations (3) and (5) collected into a column vector of dimension $(2 \mathrm{KQ}+\mathrm{K}) \times 1, \mathrm{~h}(\theta)$ is the vector of restrictions as . in equation $(6), H_{\theta}=\frac{\partial h}{\partial \theta^{T}}$ and $V(\theta)$ is the variance-covariance matrix of the unrestricted parameters all of which are evaluated at the unrestricted maximum likelihood estimates, $\hat{\theta}$. In general, this $W$ statistic would be fairly tedious to compute.

In order to derive the IM statistic, it is convenient to write equations (3) and (5) in matrix notation as: 


$$
\begin{aligned}
\mathrm{X}_{i} & =\mathrm{X}_{i}^{*} \gamma_{i}+\mathrm{u}_{i} \quad \quad i=1, \ldots, \mathrm{K} \text { equations } \\
\mathrm{Y} & =\mathrm{Z} \alpha+\epsilon
\end{aligned}
$$

where $Y$ and $X_{i}$ are $N \times 1$ vectors, $X_{i}^{*}=\left[X_{i}(1), \ldots, X_{i}(Q)\right]$ is an NXQ matrix with typical element $x_{t i}(Q)=x_{t-Q, i}, z=\left[z_{1}, \ldots, z_{K}\right]$ is an $N \times(K Q+K)$ matrix with $z_{i}=\left[X_{i}: X_{i}^{*}\right]$ an $N \times(Q+1)$ matrix, and $\gamma_{i}$ and $\alpha$ are vectors of unknown parameters of dimension $Q \times 1$ and $(K Q+K) \times 1$, respectively. In addition, we have as sumed $u_{i} \sim N\left(0, \sigma_{i}^{2} I_{N}\right), \operatorname{plim}\left(\frac{x_{i}^{* T} u_{i}}{N}\right)=0, \operatorname{plim}\left(\frac{u_{i}^{T} \epsilon}{N}\right)=0$ for all $i=1, \ldots, k$, $\operatorname{plim}\left(\frac{u_{i}^{T} u_{s}}{N}\right)=0$ for all ifs, $\epsilon \sim N\left(0, \sigma_{\epsilon}^{2} I_{N}\right)$ and $\operatorname{plim}\left(\frac{z_{\epsilon}^{T}}{N}\right)=0$. Note that

it would make no difference if $\mathrm{Z}$ had other exogenous variables in addition to the $X^{2} s$. Collecting the coefficients in $\theta$, the log likelihood function for equation ( 8 ) is:

$$
\begin{aligned}
L\left(\theta, \sigma_{1}^{2}, \ldots, \sigma_{K}^{2}, \sigma_{\epsilon}^{2}\right)= & -\frac{N(K+1)}{2} \ln 2 \pi-\frac{N}{2} \sum_{i=1}^{K} \ln \sigma_{i}^{2}-\frac{N}{2} \ln \sigma_{\epsilon}^{2} \\
& -\frac{1}{2} \sum_{i=1}^{K} \frac{1}{\sigma_{i}^{2}}\left(X_{i}-X_{i}^{*} \gamma_{i}\right)^{T}\left(X_{i}-X_{i}^{*} \gamma_{i}\right) \\
& -\frac{1}{2 \sigma_{\epsilon}^{2}}(Y-Z \alpha)^{T}(Y-Z \alpha)
\end{aligned}
$$

The IM test is obtained (see Breusch and Pagan, 1980; and Silvey, 1959) as:

$$
\tilde{D}^{T} \tilde{I}^{-1} \tilde{D} \stackrel{a}{\sim} \chi^{2}(K Q-1)
$$

under $\mathrm{H}_{\mathrm{O}}$, where "N" denotes evaluation at the restricted maximum likelihood estimates. As attention can be confined to $\theta$ in view of the block diagonality in the information matrix between $\theta$ and the $\sigma^{2}$ 's, in this case: 
$\mathrm{D}=\frac{\partial \mathrm{L}}{\partial \theta}=\left[\begin{array}{lll}\sigma_{1}^{-2} & \mathrm{x}_{1}^{* T} & \mathrm{u}_{1} \\ \sigma_{2}^{-2} & \mathrm{x}_{2}^{* T} & \mathrm{u}_{2} \\ & \vdots & \\ \sigma_{\mathrm{K}}^{-2} & \mathrm{x}_{\mathrm{K}}^{* T} & \mathrm{u}_{\mathrm{K}} \\ \sigma_{\epsilon}^{-2} & \mathrm{z}^{\mathrm{T}} & \epsilon\end{array}\right]_{(2 \mathrm{KQ}+\mathrm{K}) \times 1}$

and $\quad \begin{aligned} \quad \text { I } & =\operatorname{plim} \cdot\left[-\frac{\partial^{2} L}{\partial \theta \partial \theta^{T}}\right] \\ & =\operatorname{plim}\left[\left(\frac{\partial L}{\partial \theta}\right)\left(\frac{\partial L}{\partial \theta}\right)^{T}\right] \quad(\text { at } \theta=\tilde{\theta})\end{aligned}$

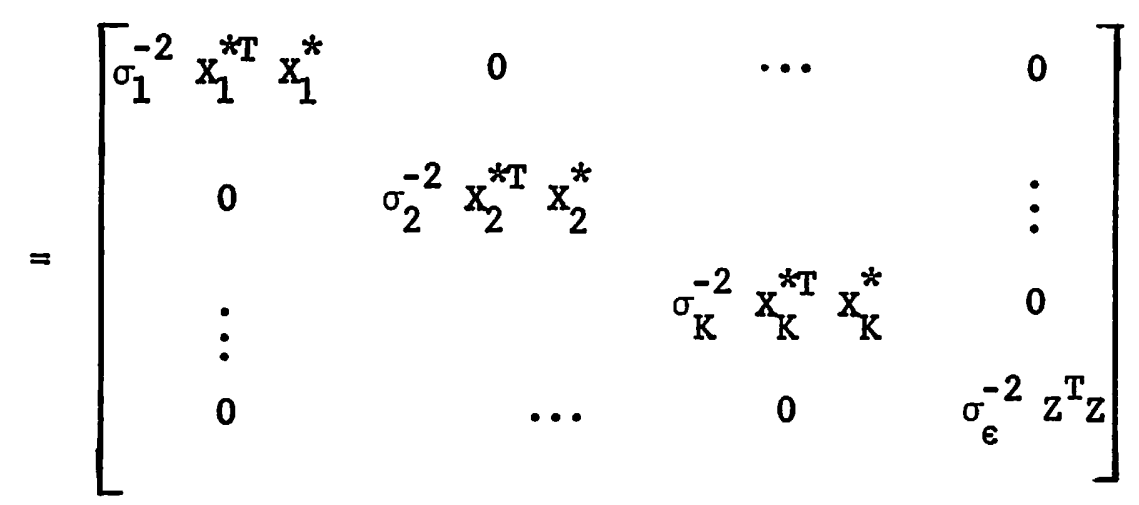

$(2 \mathrm{KQ}+\mathrm{K}) \times(2 \mathrm{KQ}+\mathrm{K})$

where the cross-product terms are zero because of the orthogonality conditions. Substitution of (11) into (10) yields:

$$
\sum_{i=1}^{K} \tilde{\sigma}_{i}^{-2} \widetilde{u}_{i}^{T} x_{i}^{*}\left(x_{i}^{* T} x_{i}^{*}\right)^{-1} x_{i}^{* T} \tilde{u}_{i}+\tilde{\sigma}_{\epsilon}^{-2} \widetilde{\epsilon}_{Z}\left(z^{T}\right)^{-1} z_{\epsilon}^{T} \approx x^{2}(K Q-1)
$$

under $\mathrm{H}_{0^{-}}$To calculate (12), simply regress the restricted residuals obtained from estimating (3) and (4) on the lagged X's appropriate to its equation, sum the (uncentered) $R^{2}$ 's for each of these $K+1$ regressions and multiply by $N$. This IM 
test presents no more computational problems than the corresponding LR statistic described earlier.

While the $W, L R$ and IM tests are in general asymptotically equivalent, in some circumstances a size ordering can be established as in Berndt and Savin (1977). While there is no complete ordering in this case, Breusch (1979) has demonstrated that when non-linear restrictions (6) are tested on a linear model (5), LR $\geq$ LM. Hence the LM test is more conservative than the $L R$ in that it tends to favour the null hypothesis, while there is no such systematic relationship involving the $W$ test.

These are two final points worth noting here. The first concerns the assumption $\operatorname{plim}\left(\frac{z^{T} \varepsilon}{N}\right)=0$. If this assumption is not made, equation (5) is not identifiable. The second regards the assumption that $\operatorname{plim}\left(\frac{u_{i}^{T} u_{s}}{N}\right)=0$ for all ifs. Relaxing this assumption would complicate the calculation of the IM test somewhat, since the equations for the exogenous variable could no longer be treated separately.

4. Monte Carlo results

Monte Carlo analysis was used to examine the small sample properties of the test. To facilitate comparison with $\mathrm{H}-\mathrm{S}$, the experiment follows their design exactly, except that the LM test is computed as well as the W and LR. For the cases where the rational expectations restrictions are true, the X's and $y^{\prime}$ s were generated from (3) and (4) respectively using a random normal deviate generator and selected values of the following parameters: sample size (N); number of exogenous variables (K); order of the autoregressive process $(Q), \beta$; autoregressive parameters $(\gamma), \lambda, \sigma_{i}^{2}(i=1, \ldots, K)$ and $\sigma_{\epsilon}^{2}$. 
For each set of parameters, 1000 different data sets were created and the $W$, LR and LM test statistics were calculated for each. Table 1 gives the number of rejections (at the $0.01,0.05$ and 0.10 percent significance levels) and the mean and variance for each test statistic over the 1000 iterations.

Case 1 in table 1 sets $K=2, Q=1, \beta_{1}=\beta_{2}=1, \gamma_{1}=\gamma_{2}=0.8$, $\lambda=0.5$ and $\sigma_{1}=\sigma_{2}=\sigma_{\epsilon}=1$. For the remaining cases these parameters are altered as noted at the bottom of the table. The basic result is that except for Cases 9 and 10, the three tests yield about identical results. This confirms the H-S Monte Carlo results concerning the $W$ and LR tests and shows that even though the LM statistic must be numerically smaller than the IR (and hence must reject less often), the differences are typically small for the range of parameter values considered. ${ }^{3}$ While the LM can be greater or less than the $W$, in these cases it usually rejects slightly less often while there is no apparent systematic relationship between the IR and $W$. Al1 tests perform reasonably well for $\mathrm{N} \geq 100$ but there is some tendency to reject too frequently in small samples. 4

For Cases 9 and 10 the variance of the structural equations is set much greater than the variance of the autoregressions and hence they are probably unlike most actual applications (as noted by H-S). For these cases the LM test still gives very similar results to the LR but the $W$ statistic tends to be somewhat smaller than both, although the size of this difference tends to shrink for larger $\mathrm{N}$.

In Table 2, the power of the test under several alternative hypotheses is considered. For Cases 21-24, the y's are generated using the parameter values of case 1 except $\alpha_{11}=0.0,0.2,0.4$ and 0.6 respectively. The rational expectations restrictions imply an $\alpha_{11}$ of 0.8 and therefore fail with the 


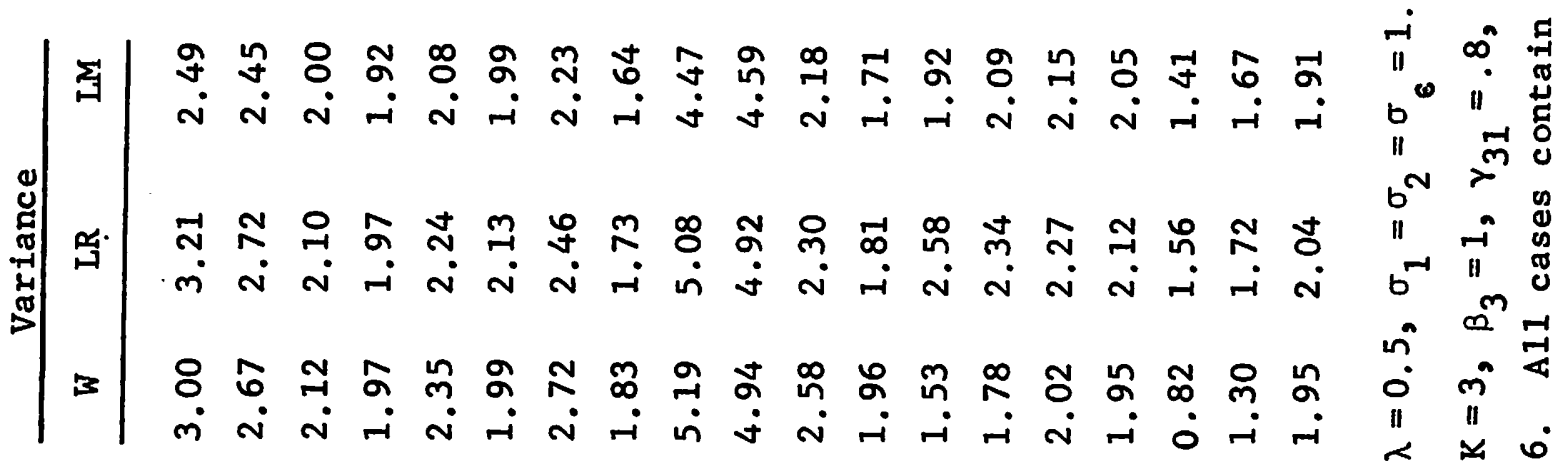

สิ $ส$ ส

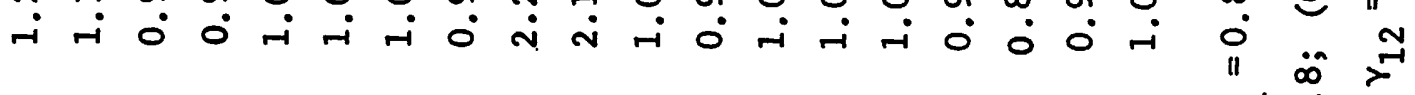
$\vec{m} \leadsto$ の

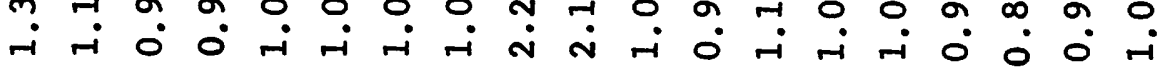

๗ 구 点

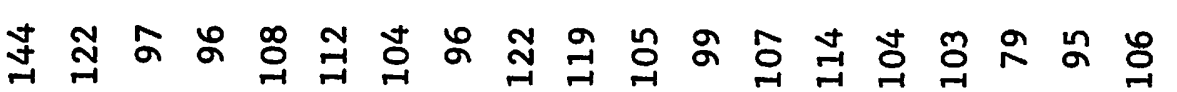

"n "N

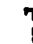

|

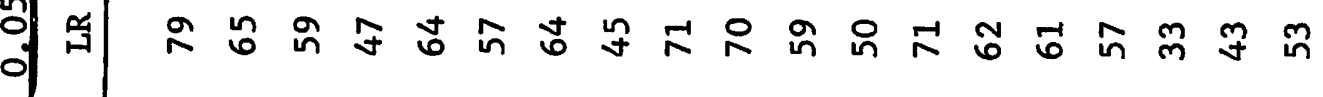
น้ సี่

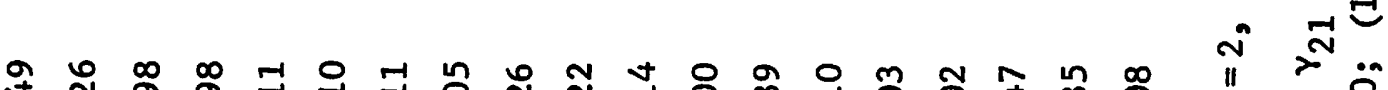
ป స్

3 ×

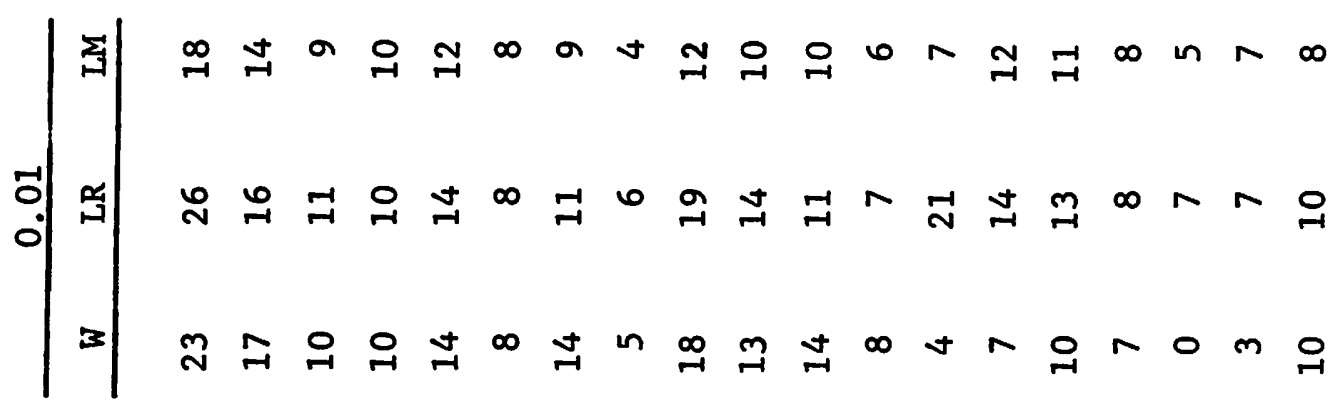

Е $\ddot{i} b^{\infty}$

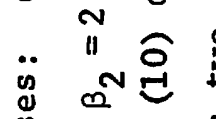

ญ

$a_{n} d$

बं

0

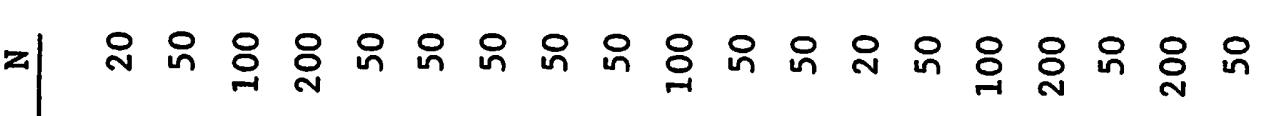
Ð $\sim m+n \infty \infty$ 우구 


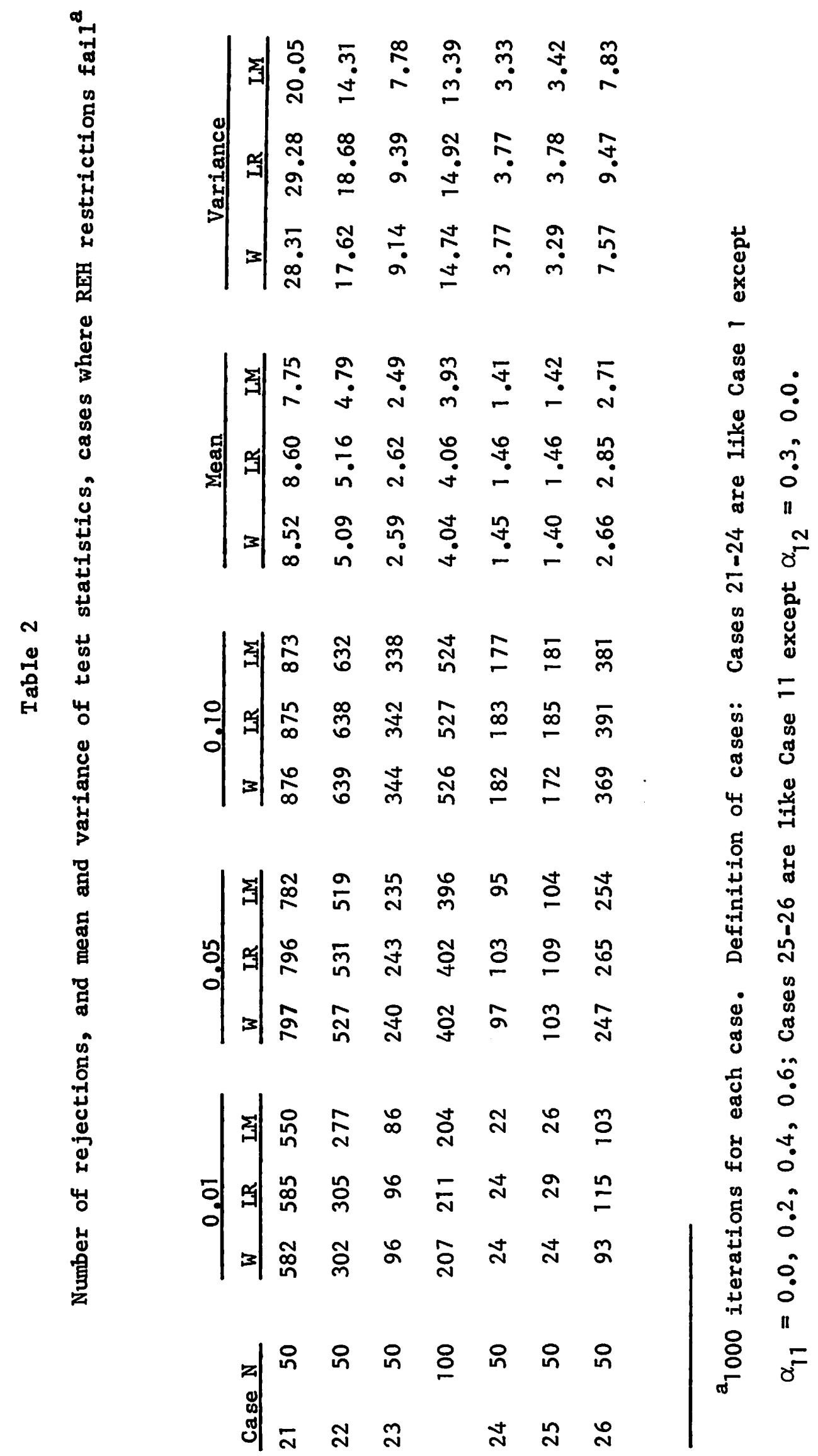


amount of failure decreasing from Cases 21 to 24 . Cases 25 and 26 are identical to Case 11 with $\alpha_{12}=0.3$ and 0.0 respectively (compared to 0.6 under rational expectations). For all these cases the three tests give very similar results. For Cases 21 to 24 , the LM tends to have the fewest rejections of the three tests while for Cases 25 and 26, the LM rejects slightly more often than the $W$, although of course never as often as the IR.

We also investigated some examples of the power against the adaptive expectations alternative of $\mathrm{H}-\mathrm{S}$ (see their Table 3 and $\mathrm{pp}, 280-282$ ). Our results (not shown but available from the authors on request) were that the LM test again performed very similarly to the two other tests.

5. Conclusions

It has been shown that the Iagrange multiplier test of the parameter restrictions implied by a simple but common class of rational expectations models can be computed as the sum of the $\mathrm{R}^{2}$ 's from a set of auxiliary regressions involving the residuals from restricted estimation. Therefore the LM test may be used as an alternative or in conjunction with the likelihood ratio and Wald tests. In particular, a researcher who has calculated the likelihood ratio (and hence has the restricted estimates) can easily compute the corresponding Lagrange multiplier statistic, which must be smaller and hence be more conservative in the rejection of the null hypothesis.

However, while this may be a worthwhile procedure, Monte Carlo evidence presented above suggests that the LM statistic is likely to be close to both the LR and $W$ statistics, even in fairly small samples. Hence in practice, the three tests are likely to perform very similarly. 


\section{FOOINOTES}

${ }^{1}$ Although there are several specific rational expectations models in this class (see, for example, Attfield et al, 1981 and Leiderman, 1980), the H-S formulation is more general and quite convenient for our analysis.

2 These assumptions are quite strict (compare Hansen and Sargent, 1982) but simplify the estimation problem.

3t should be noted that for small samples, the W-test is sensitive to the manner in which the nonlinear restrictions are specified. Originally, the authors followed the form of equation (6) and in some cases obtained remarkably different results from $\mathrm{H}-\mathrm{S}$, even for $\mathrm{N} \geq 100$. Subsequent examination of the $\mathrm{H}-\mathrm{S}$ computer program, kindly supplied by Professor Schmidt, indicated that the null hypothesis was tested in the form $H_{0}: \alpha_{i j} \alpha_{m o} \gamma_{\operatorname{mm}}-\alpha_{m n} \alpha_{i o} \gamma_{i j}=0, i, m=1, \ldots, k$ and $j, n=1, \ldots, Q$. For the results presented here we have used this form. Obviously for large $\mathrm{N}$ where the restrictions are true, the manner in which the nuIl is specified is irrelevant.

${ }^{4}$ Another alternative test uses (7) but replaces the asymptotic covariance matrix $\hat{v}$ with one adjusted for degrees of freedom (as in a standard ordinary least squares covariance matrix). The Monte Carlo experiment indicated that this test typically came closer to the correct number of rejections than the other tests for $\mathrm{N}=20$ or $\mathrm{N}=50$ but of course the adjustment made little difference for large $N$. 


\section{REFERENCES}

Attfield, C. L. F., D. Demery and N. W. Duck, 1981, A quarterly model of unanticipated monetary growth and the price level in the U.K. 1963-1978, Journal of Monetary Economics 8, 331-350.

Berndt, E. R. and N. E. Savin, 1977, Conflict among criteria for testing hypotheses in the multivariate linear regression model, Econometrica 45, 1263-1278.

Breusch, T. S., 1979, Conflict among criteria for testing hypotheses: extensions and comments, Econometrica 47, 203-207.

Breusch, T. S. and A. R. Pagan, 1980, The Lagrange multiplier test and its applications to model specifications in econometrics, Review of Economic Studies 47, 239-253.

Hansen, I. P. and T. J. Sargent, 1982, Instrumental variables procedures for estimating linear rational expectations models, Journal of Monetary Economics, forthcoming.

Hoffman, D. L. and S. Schmidt, 1981, Testing the restrictions implied by the rational expectations hypothesis, Journal of Econometrics 15, 265-287.

Leiderman, L., 1980, Macroeconometric testing of rational expectations and structure neutrality hypotheses for the United States, Journal of Monetary Economics 6, 69-82.

Revankar, N. S., 1980, Testing of the rational expectations hypothesis, Econometrica 48, 1347-1363.

Silvey, S. D., 1959, The Lagrange multiplier test, Annals of Mathematical Statistics $30,389-407$.

Wallis, K. F., 1980, Econometric implications of the rational expectations hypothesis, Econometrica 48, 49-73. 